Article

\title{
On the Fredholm Property of the Trace Operators Associated with the Elastic Layer Potentials
}

\author{
Giulio Starita and Alfonsina Tartaglione *(D) \\ Department of Mathematics and Physics, University of Campania, 81100 Caserta, Italy; giulio.starita@unicampania.it \\ * Correspondence: alfonsina.tartaglione@unicampania.it
}

Received: 21 December 2018; Accepted: 24 January 2019; Published: 1 February 2019

\begin{abstract}
We deal with the system of equations of linear elastostatics, governing the equilibrium configurations of a linearly elastic body. We recall the basics of the theory of the elastic layer potentials and we extend the trace operators associated with the layer potentials to suitable sets of singular densities. We prove that the trace operators defined, for example, on $W^{1-k-1 / q, q}(\partial \Omega)$ (with $k \geq 2, q \in(1,+\infty)$ and $\Omega$ an open connected set of $\mathbb{R}^{3}$ of class $\left.C^{k}\right)$, satisfy the Fredholm property.
\end{abstract}

Keywords: linear elastostatics; layer potentials; fredholmian operators

MSC: 74B05; 35Q74; 45B05

\section{Introduction}

As is well-known [1], the equilibrium configurations of a homogeneous linearly elastic body $\Omega \subset \mathbb{R}^{3}$ (see Notation and Functional spaces in Section 2) with no body forces acting on it, satisfy the differential system

$$
\operatorname{div} \mathbb{C}[\nabla \boldsymbol{u}]=\mathbf{0},
$$

where $\mathbb{C}$ is the elasticity tensor and $u$ is the unknown displacement field. Wide efforts have been directed, from a theoretical point of view, to the problem of existence and uniqueness of solutions of system (1) when the displacement, the traction, or a combination of them are prescribed on the boundary (see, e.g., [2-5]). In all the cited references, the regularity of the boundary values is required, since the problem is formulated within the approach of the variational theory. Nevertheless, in view of possible applications, it is clear that the investigation of the boundary value problems when the data are singular is a notable and engaging issue. Now, since the elasticity tensor $\mathbb{C}$ is independent on the point, the analysis can be done by means of the elastic layer potentials defined through the fundamental solution (see Section 2). In particular, the proof of the existence and uniqueness of a solution of (1) passes through the possibility to apply the Fredholm alternative to the integral equation translating the boundary value problem which is examined. So, a preliminary step in the analysis of the existence and uniqueness problem is to show that the trace operators involved in the integral equations satisfy the so-called Fredholm property (see Notation and Functional spaces in Section 2). Obviously, this is well-understood when the densities are regular fields on the boundary (see, e.g., [6]). The aim of this paper is to show that the Fredholm property is also met for singular densities. For example, we prove that the trace operator associated with the single layer potential with density in $W^{1-k-1 / q, q}(\partial \Omega)$ is Fredholmian.

The paper is organized as follows. In Section 2 we recall some classical results about the system of homogeneous elastostatics and some notations on the involved functional spaces. In Sections 3 and 4 we 
recall the most important facts about the layer potentials and we prove the Fredholm property for the associated trace operators.

\section{Some Classical Results of Homogeneous Elastostatics}

We essentially follow the notation in [1]. In particular, we denote by Lin the set of all tensors, i.e., linear applications from $\mathbb{R}^{3}$ to $\mathbb{R}^{3}$ and by $\mathrm{Skw} \subset$ Lin the set of all skew tensors. We use bold lower-case letters, like $\boldsymbol{a}$ and $\boldsymbol{b}$, for vectors, and bold upper-case letters, like $\boldsymbol{E}, \boldsymbol{L}$ and $\boldsymbol{W}$ for tensors.

Recall that the elasticity tensor $\mathbb{C}$, representing the elastic properties of the body, is a linear map from Lin $\rightarrow$ Lin such that

$$
\mathbb{C}[\boldsymbol{W}]=\mathbf{0}, \forall \boldsymbol{W} \in \mathrm{SkW}
$$

and

$$
\boldsymbol{E} \cdot \mathbb{C}[\boldsymbol{L}]=\boldsymbol{L} \cdot \mathbb{C}[\boldsymbol{E}], \quad \forall \boldsymbol{E}, \boldsymbol{L} \in \mathrm{Lin}
$$

$\mathbb{C}$ is positive definite if

$$
\pi[\boldsymbol{E}] \geq|\operatorname{sym} E|^{2}, \quad \forall \boldsymbol{E} \in \operatorname{Lin}
$$

where

$$
\pi[\boldsymbol{E}]=\boldsymbol{E} \cdot \mathbb{C}[\boldsymbol{E}], \quad \forall \boldsymbol{E} \in \operatorname{Lin}
$$

and $\mathbb{C}$ is strongly elliptic if

$$
\pi[\boldsymbol{a} \otimes \boldsymbol{b}]=(\boldsymbol{a} \otimes \boldsymbol{b}) \cdot \mathbb{C}[\boldsymbol{a} \otimes \boldsymbol{b}]>\mathbf{0}, \quad \forall \boldsymbol{a}, \boldsymbol{b} \neq \mathbf{0} .
$$

- $\quad$ From now on we shall assume $\mathbb{C}$ to be at least strongly elliptic.

A weak solution of (1) (variational solution for $q=2$ ) is a field $u \in W_{\text {loc }}^{1, q}(\Omega)$ such that

$$
\int_{\Omega} \nabla \boldsymbol{\phi} \cdot \mathbb{C}[\nabla \boldsymbol{u}]=0, \quad \forall \boldsymbol{\phi} \in C_{0}^{\infty}(\Omega) .
$$

It is well-known that for $\mathbb{C}$ strongly elliptic, every weak solution to (1) is analytical in $\Omega$.

Equation (1) admits a fundamental solution $\boldsymbol{U}(x-y)$ [7], i.e., a regular solution for all $x \neq y$ to

$$
\operatorname{div} \mathbb{C}[\nabla \boldsymbol{U}(x-y)]=\delta(x-y)
$$

where $\delta$ denotes the Dirac distribution, expressed by

$$
U(z)=\frac{\Phi(z)}{|z|},
$$

with $\boldsymbol{\Phi}$ homogeneous second-order tensor function of degree zero.

If $\Omega$ is a bounded domain, then a standard computation assures that every solution $u \in W^{1, q}(\Omega)$ of (1) is represented by the Somigliana formula [1]

$$
\boldsymbol{u}(x)=\int_{\partial \Omega}^{\star} \boldsymbol{U}(x-\zeta) \boldsymbol{s}(\boldsymbol{u})(\zeta) \mathrm{d} \sigma_{\zeta}+\int_{\partial \Omega}^{\star} \mathbb{C}[\nabla \boldsymbol{U}(x-\zeta)](\boldsymbol{u} \otimes \boldsymbol{n})(\zeta) \mathrm{d} \sigma_{\zeta}
$$

for all $x \in \Omega$, where

$$
\boldsymbol{s}(\boldsymbol{u})=\mathbb{C}[\nabla \boldsymbol{u}] \boldsymbol{n} \quad \text { on } \partial \Omega
$$


is the traction field on $\partial \Omega$ associated with $\boldsymbol{u}$ (from now on we denote by $\boldsymbol{n}$ the unit normal to $\partial \Omega$ exterior [resp. interior] with respect to $\Omega$ for $\Omega$ bounded [resp. exterior] domain). Starting from (9) and making use of Liouville's theorem (see, e.g., [8,9]) one proves that if $\boldsymbol{u} \in W_{\mathrm{loc}}^{1, q}(\bar{\Omega})$ is a solution of (1) in an exterior domain such that $\boldsymbol{u}=o\left(r^{2}\right)$, then (9) becomes

$$
\begin{aligned}
\boldsymbol{u}(x) & =\boldsymbol{A} \boldsymbol{x}+\boldsymbol{u}_{0}+\int_{\partial \Omega}^{\star} \boldsymbol{U}(x-\zeta) \boldsymbol{s}(\boldsymbol{u})(\zeta) \mathrm{d} \sigma_{\zeta} \\
& +\int_{\partial \Omega}^{\star} \mathbb{C}[\nabla \boldsymbol{U}(x-\zeta)](\boldsymbol{u} \otimes \boldsymbol{n})(\zeta) \mathrm{d} \sigma_{\zeta},
\end{aligned}
$$

for suitable constants $\boldsymbol{u}_{0}$ and $A$. Hence the following representation follows

$$
u(x)=A x+u_{0}+U(x) \int_{\partial \Omega}^{\star} s(u)+f(x)
$$

with

$$
\nabla_{k} f(x)=O\left(r^{-2-k}\right)
$$

Clearly, for $A=u_{0}=\mathbf{0}$,

$$
\boldsymbol{u}=O\left(r^{-2}\right) \Leftrightarrow \int_{\partial \Omega}^{\star} \boldsymbol{s}(\boldsymbol{u})=\mathbf{0} .
$$

Let $u \in W_{\text {loc }}^{1,2}(\bar{\Omega})$ be a variational solution of (1). If $\Omega$ is bounded, then the work and energy theorem follows [1]

$$
\int_{\Omega} \pi[\nabla \boldsymbol{u}]=\int_{\partial \Omega}^{\star} \boldsymbol{u} \cdot \boldsymbol{s}(\boldsymbol{u})
$$

Let denote by $\mathfrak{R}$ the set of all (infinitesimal) rigid displacements.

If $\Omega$ is exterior and $\boldsymbol{u}=\boldsymbol{\varrho}+o(1)$, with $\boldsymbol{\varrho}=\boldsymbol{u}_{0}+\boldsymbol{\omega} \times \boldsymbol{x} \in \mathfrak{R}$ assigned, (13) implies

$$
\int_{\Omega_{R}} \pi[\nabla \boldsymbol{u}]=\int_{\partial \Omega}^{\star}(\boldsymbol{u}-\boldsymbol{\varrho}) \cdot \boldsymbol{s}(\boldsymbol{u})+\int_{\partial S_{R}}(\boldsymbol{u}-\boldsymbol{\varrho}) \cdot \boldsymbol{s}(\boldsymbol{u}) .
$$

Hence, taking into account that by (11) $(\boldsymbol{u}-\boldsymbol{\varrho}) \cdot \boldsymbol{s}(\boldsymbol{u})=O\left(r^{-3}\right)$, letting $R \rightarrow+\infty$ we obtain the work and energy theorem in exterior domains [1]

$$
\int_{\Omega} \pi[\nabla u]=\int_{\partial \Omega}^{\star} u \cdot s(u)-u_{0} \cdot \int_{\partial \Omega}^{\star} s(u)-\omega \cdot \int_{\partial \Omega}^{\star} x \times s(u) .
$$

The following result is due to L. Van Hove [10] (see also [1] p. 105).

Lemma 1. It holds

$$
\int_{\Omega}|\nabla \boldsymbol{u}|^{2} \leq \int_{\Omega} \pi[\nabla \boldsymbol{u}]
$$

for all $\boldsymbol{u} \in D_{0}^{1,2}(\Omega)$, where $D_{0}^{1,2}(\Omega)$ denotes the completion of $C_{0}^{\infty}(\Omega)$ with respect to $\|\nabla \boldsymbol{\phi}\|_{L^{2}(\Omega)}$.

Relations (13), (14) and Lemma 1 imply the following classical uniqueness results [1]: if $\boldsymbol{u}$ is a variational solution of (1), with $\boldsymbol{u}=o(1)$ for $\Omega$ exterior, then

$$
u_{\mid \partial \Omega}=0 \Rightarrow u \equiv 0
$$


and

$$
\mathbb{C} \text { positive definite, } \quad \int_{\partial \Omega}^{\star} \boldsymbol{u} \cdot \boldsymbol{s}(\boldsymbol{u}) \leq 0 \Longrightarrow \boldsymbol{u} \begin{cases}\in \mathfrak{R}, & \Omega \text { bounded } \\ =\mathbf{0}, & \Omega \text { exterior. }\end{cases}
$$

- From now on uniqueness for the traction problem in bounded domains will be understood in the class of normalized displacement, i.e, the set of fields $u$ such that (cf. [1] p. 110)

$$
\int_{\partial \Omega}^{\star} u=0, \quad \int_{\partial \Omega}^{\star} x \times u=0 .
$$

We will need the following result.

Lemma 2. If $\boldsymbol{u}$ is a variational solution of (1), then

$$
\|\boldsymbol{u}\|_{W^{k, 2}\left(\Omega^{\prime \prime}\right)} \leq c\|\boldsymbol{u}\|_{L^{2}(\tilde{\Omega})}
$$

for all bounded domains $\Omega^{\prime \prime}, \tilde{\Omega}$ such that $\overline{\Omega^{\prime \prime}} \subset \tilde{\Omega} \subset \Omega$, with c independent of $\boldsymbol{u}$.

Proof. (15) is a simple consequence of the classical Caccioppoli's inequality (see, e.g., [11])

$$
\int_{S_{R}\left(x_{0}\right)}|\nabla \boldsymbol{u}|^{2} \leq \frac{c}{R^{2}} \int_{S_{2 R}\left(x_{0}\right) \backslash S_{R}\left(x_{0}\right)}|\boldsymbol{u}|^{2}
$$

for all $x_{0} \in \Omega$ and $R<\operatorname{dist}\left(x_{0}, \partial \Omega\right) / 2$, with $c$ independent of $\boldsymbol{u}$, taking into account that any derivative of $\boldsymbol{u}$ is a solution of (1) and making use of Sobolev's lemma.

Notation and Functional spaces - The body is identified with the domain $\Omega \subset \mathbb{R}^{3}$ it occupies in a reference configuration. We suppose $\Omega$ to be a bounded or exterior domain of class $C^{k}(k \geq 2)$. We denote by $o$ the origin of the reference frame; we suppose $o \in \Omega$ [resp. $o \in[\bar{\Omega}$ ] for $\Omega$ bounded [resp. exterior] domain. For every $x \in \mathbb{R}^{3}$ we set $x=x-o ; r=|x|$. Unless otherwise specified, in the formulas including integrals, the variable of integration is a point of the region indicated by the integral $(\Omega, \partial \Omega$, etc.); we shall omit it when it will be clear from the context. If $\Omega$ is exterior, we set $\Omega_{R}=\Omega \cap S_{R}$, where $S_{R}=\left\{x \in \mathbb{R}^{3}: r<R\right\}$ and, as usual, if $f(x)$ and $g(r)>0$ are two functions on $\Omega$, by $f=o(g)$ and $f=O(g)$ we mean that $\lim _{r \rightarrow+\infty} f(x) / g(r)=0$ and $|f(x)| \leq c g(r)$.

$W^{k, q}(\Omega)$ is the Sobolev space of all $\varphi \in L_{\text {loc }}^{1}(\Omega)$ such that $\|\varphi\|_{W^{k, q}(\Omega)}=\|\varphi\|_{L^{q}(\Omega)}+\left\|\nabla_{k} \varphi\right\|_{L^{q}(\Omega)}<$ $+\infty ; W_{0}^{k, q}(\Omega)$ is the completion of $C_{0}^{\infty}(\Omega)$ with respect to $\|\varphi\|_{W^{k, q}(\Omega)}$ and $W^{-k, q^{\prime}}(\Omega)$ is its dual space. $W^{k-1 / q, q}(\partial \Omega)$ is the trace space of $W^{k, q}(\Omega)$ and $W^{1-k-1 / q^{\prime}, q^{\prime}}(\partial \Omega)$ is its dual space. We set

$$
\int_{\Omega}^{\star} f \varphi \quad\left[\int_{\partial \Omega}^{\star} f \varphi\right]
$$

to denote (say) the value of the functional $f \in W^{-k, q^{\prime}}(\Omega)\left[f \in W^{-k, q^{\prime}}(\partial \Omega)\right]$ at $\varphi \in W_{0}^{k, q}(\Omega)\left[\varphi \in W^{k, q}(\partial \Omega)\right]$. Of course, if $f \varphi$ is integrable, then $\int \equiv \int^{\star}$. If $\Omega$ is of class $C^{k}$, since $W^{k-1 / q, q}(\partial \Omega) \hookrightarrow C^{k-1, \mu}(\partial \Omega)$, for $k q>3$ and $\mu=1-3 / q$, we have that $\left[C^{k-1, \mu}(\partial \Omega)\right]^{\prime} \hookrightarrow W^{1-k-1 / q^{\prime}, q^{\prime}}(\partial \Omega)$. Then, in particular, $W^{-1, q}(\partial \Omega), q \in(1,2)$ contains the space of all Borel measures on $\partial \Omega$.

If $\mathcal{F}(\Omega)$ is a functional space in $\Omega$, we denote by $\mathcal{F}_{\text {loc }}(\Omega)$ the set of all functions that belong to $\mathcal{F}(K)$ for every compact set $K \subset \Omega$. 
Let $\mathcal{B}, \mathcal{D}$ be two Banach spaces and denote by $\mathcal{B}^{\prime}, \mathcal{D}^{\prime}$ their dual spaces. A linear and continuous map $\mathcal{T}: \mathcal{B} \rightarrow \mathcal{D}$ is said to be Fredholmian (or satisfies the Fredholm property) if its range is closed and $\operatorname{dim} \operatorname{Kern} \mathcal{T}=\operatorname{dim} \operatorname{Kern} \mathcal{T}^{\prime} \in \mathbb{N}_{0}$, where $\mathcal{T}^{\prime}: \mathcal{D}^{\prime} \rightarrow \mathcal{B}^{\prime}$ is the adjoint of $\mathcal{T}$. A Fredholmian operator satisfies the classical Fredholm alternative and a well-known result of J. Peetre [12] assures that $\mathcal{T}$ is Fredholmian if there is a compact operator $\mathcal{C}$ from $\mathcal{B}$ into a Banach space $\mathcal{G}$ such that

$$
\|u\|_{\mathcal{B}} \leq c\left\{\|\mathcal{T}[u]\|_{\mathcal{D}}+\|\mathcal{C}[u]\|_{\mathcal{G}}\right\}
$$

and $\operatorname{dim} \operatorname{Kern} \mathcal{T}=\operatorname{dim} \operatorname{Kern} \mathcal{T}^{\prime}$.

\section{The Trace Operators Associated with the Simple Layer Potential}

Every integral at right hand side of (9) is an analytic solution of (1) in $\mathbb{R}^{3} \backslash \partial \Omega$. More in general, for every $\psi \in L^{1}(\partial \Omega)$ the field

$$
\boldsymbol{v}[\boldsymbol{\psi}](x)=\int_{\partial \Omega} \boldsymbol{U}(x-\zeta) \boldsymbol{\psi}(\zeta) \mathrm{d} \sigma_{\zeta}
$$

defines an analytical solution of (1) in $\mathbb{R}^{3} \backslash \partial \Omega$ known as simple layer potential with density $\psi$. Note that $v[\psi]$ behaves at infinity as the fundamental solution $U$. In particular,

$$
\nabla_{k} v[\boldsymbol{\psi}](x)=O\left(r^{-1-k}\right)
$$

and

$$
\int_{\partial \Omega} \boldsymbol{\psi}=\mathbf{0} \Rightarrow \nabla_{k} \boldsymbol{v}[\boldsymbol{\psi}](x)=O\left(r^{-2-k}\right) .
$$

It is well-known that for a density $\psi \in W^{k-1-1 / q, q}(\partial \Omega)$

$$
\|\boldsymbol{v}[\boldsymbol{\psi}]\|_{W^{k, q}(\Omega)} \leq c\|\boldsymbol{\psi}\|_{W^{k-1-1 / q, q(\partial \Omega)}}
$$

with $c$ independent of $\psi$, the limit

$$
\lim _{\epsilon \rightarrow 0^{ \pm}} v[\boldsymbol{\psi}](\xi-\epsilon \boldsymbol{l}(\xi))=\mathcal{S}[\boldsymbol{\psi}](\xi)
$$

exists for almost all $\xi \in \partial \Omega$ (by the embedding theorem if $q>3 / k$, then (20) holds for all $\xi$ ) and axis $l$ in a ball tangent (on the side of $n$ ) to $\partial \Omega$ at $\xi$ and defines the trace of the simple layer potential with density $\psi[13]$. As a consequence, $v[\psi]$ is continuous in $\mathbb{R}^{3}$. Moreover, the map

$$
\mathcal{S}: W^{k-1-1 / q, q}(\partial \Omega) \rightarrow W^{k-1 / q, q}(\partial \Omega)
$$

is continuous; accordingly,

$$
\|\mathcal{S}[\boldsymbol{\psi}]\|_{W^{k-1 / q, q(\partial \Omega)}} \leq \mathcal{c}\|\psi\|_{W^{k-1-1 / q, q(\partial \Omega)}}
$$

for some constant $c$ depending only on $k, q$ and $\Omega$. Let $\psi \in W^{1-k-1 / q, q}(\partial \Omega)$ and let $\psi_{k}$ be a regular sequence which converges to $\psi$ strongly in $W^{1-k-1 / q, q}(\partial \Omega)$. By (22)

$$
\left|\int_{\partial \Omega} \boldsymbol{\phi} \cdot \mathcal{S}\left[\boldsymbol{\psi}_{k}\right]\right|=\left|\int_{\partial \Omega} \boldsymbol{\psi}_{k} \cdot \mathcal{S}[\boldsymbol{\phi}]\right| \leq c\left\|\boldsymbol{\psi}_{k}\right\|_{W^{1-k-1 / q, q(\partial \Omega)}}\|\boldsymbol{\phi}\|_{W^{k-1-1 / q, q(\partial \Omega)}} .
$$

Therefore, by well-known results of functional analysis, $\mathcal{S}$ can be extended to a linear and continuous operator

$$
\mathcal{S}^{\prime}: W^{1-k-1 / q^{\prime}, q^{\prime}}(\partial \Omega) \rightarrow W^{2-k-1 / q^{\prime}, q^{\prime}}(\partial \Omega)
$$


which is the adjoint of $\mathcal{S}$ and defines the trace of the simple layer with density $\psi \in W^{1-k-1 / q^{\prime}, q^{\prime}}(\partial \Omega)$ :

$$
\boldsymbol{v}[\boldsymbol{\psi}](x)=\int_{\partial \Omega}^{\star} \boldsymbol{U}(x-\zeta) \boldsymbol{\psi}(\zeta) \mathrm{d} \sigma_{\zeta}
$$

By (19) it is not difficult to see that

$$
\|\boldsymbol{v}[\boldsymbol{\psi}]\|_{W^{2-k, q^{\prime}}(\Omega)} \leq c\|\psi\|_{W^{1-k-1 / q^{\prime}, q^{\prime}}(\partial \Omega)} .
$$

The traction field associated with the simple layer potential (16) with density $\psi \in W^{k-1-1 / q, q}(\partial \Omega)$ is defined on both "faces" of $\partial \Omega$ by the limit

$$
\lim _{\epsilon \rightarrow 0^{+}} \mathbb{C}[\nabla \boldsymbol{v}[\boldsymbol{\psi}]](\xi \mp \epsilon \boldsymbol{l}(\xi)) \boldsymbol{n}(\xi)=\mathcal{T}^{ \pm}[\boldsymbol{\psi}](\xi)
$$

for almost all $\xi \in \partial \Omega$ (by the embedding theorem if $q>3 /(k-1)$, then (25) holds for all $\xi$ ) and axis $l$ in a ball tangent (on the side of $n$ ) to $\partial \Omega$ at $\xi$. Moreover,

$$
\left\|\mathcal{T}^{ \pm}[\boldsymbol{\psi}]\right\|_{W^{k-1-1 / q, q(\partial \Omega)}} \leq c\|\boldsymbol{\psi}\|_{W^{k-1-1 / q, q(\partial \Omega)}}
$$

for some constant $c$ depending only on $k, q$ and $\Omega$, and the classical jump condition holds

$$
\psi=\mathcal{T}^{+}[\psi]-\mathcal{T}^{-}[\boldsymbol{\psi}]
$$

We now show that the trace operator $\mathcal{S}$ is Fredholmian. To this aim we make use of the following well-known results (cf. [11,14-18]).

Lemma 3. Let $\Omega$ be a bounded domain of class $C^{k}(k \geq 2)$. If $\hat{\boldsymbol{u}} \in W^{k-1 / q, q}(\partial \Omega), q \in(1,+\infty)$, and $\boldsymbol{\phi} \in C_{0}^{\infty}(\Omega)$, then the displacement problem

$$
\begin{aligned}
\operatorname{div} \mathbb{C}[\nabla \boldsymbol{u}]=\boldsymbol{\phi} & \text { in } \Omega, \\
\boldsymbol{u}=\hat{\boldsymbol{u}} & \text { on } \partial \Omega,
\end{aligned}
$$

has a unique solution $\boldsymbol{u} \in W^{k, q}(\Omega)$ and

$$
\|\boldsymbol{u}\|_{W^{k, q}(\Omega)} \leq \mathcal{c}\left\{\|\hat{\boldsymbol{u}}\|_{W^{k-1 / q, q(\partial \Omega)}}+\|\boldsymbol{\phi}\|_{W^{k-2, q}(\Omega)}\right\} .
$$

Lemma 4. Let $\Omega$ be a bounded domain of class $C^{k}(k \geq 2)$. If $\hat{s} \in W^{k-1-1 / q, q}(\partial \Omega), q \in(1,+\infty)$ satisfies

$$
\int_{\partial \Omega} \varrho \cdot \hat{s}=0, \quad \forall \varrho \in \Re
$$

and $\boldsymbol{\phi} \in C_{0}^{\infty}(\Omega)$, then the traction problem

$$
\begin{array}{rlrl}
\operatorname{div} \mathbb{C}[\nabla \boldsymbol{u}] & =\boldsymbol{\phi} & & \text { in } \Omega, \\
\boldsymbol{s}(\boldsymbol{u})=\hat{\boldsymbol{s}} & & \text { on } \partial \Omega,
\end{array}
$$

has a unique normalized solution $\boldsymbol{u} \in W^{k, q}(\Omega)$ and

$$
\|\boldsymbol{u}\|_{W^{k, q}(\Omega)} \leq c\left\{\|\hat{\boldsymbol{s}}\|_{W^{k-1-1 / q, q(\partial \Omega)}}+\|\boldsymbol{\phi}\|_{W^{k-2, q}(\Omega)}\right\} .
$$

The following theorem holds true. 
Theorem 1. Let $\Omega$ be a bounded or an exterior domain of class $C^{k}(k \geq 2), k \in \mathbb{N}$. The operator $\mathcal{S}$ is Fredholmian and $\operatorname{Kern} \mathcal{S}=\operatorname{Kern} \mathcal{S}^{\prime}=\{\mathbf{0}\}$.

Proof. Let $\Omega$ be bounded. By the trace theorem, (20) and classical interior estimates (see Lemma 2) from (27) it follows

$$
\begin{aligned}
\|\boldsymbol{\psi}\|_{W^{k-1-1 / q, q}(\partial \Omega)} & \leq\left\|\mathcal{T}^{+}[\boldsymbol{\psi}]\right\|_{W^{k-1-1 / q, q}(\partial \Omega)}+\left\|\mathcal{T}^{-}[\boldsymbol{\psi}]\right\|_{W^{k-1-1 / q, q}(\partial \Omega)} \\
& \leq c\left\{\|\boldsymbol{v}[\boldsymbol{\psi}]\|_{W^{k, q}(\Omega)}+\|\boldsymbol{v}[\boldsymbol{\psi}]\|_{W^{k, q}\left(C \Omega \cap S_{R}\right)}\right\} \\
& \leq c\left\{\| \mathcal{S}\left[\boldsymbol{\psi}\left\|_{W^{k-1 / q, q}(\partial \Omega)}+\right\| \mathcal{C} \|\right\}\right.
\end{aligned}
$$

where $S_{R}(\supset \bar{\Omega})$ is a ball of radius $R$ centered at $o$ and $\mathcal{C}$ is a completely continuous map from $W^{k-1-1 / q, q}(\partial \Omega)$ in a Banach space. Hence by Peetre's result (see Notation and Functional spaces in Section 2) it follows that $\mathcal{S}$ has a closed range. If $\psi \in \operatorname{Kern} \mathcal{S}$ then by (17) an integration by parts gives

$$
\begin{aligned}
& \int_{\Omega} \pi[\nabla \boldsymbol{v}[\boldsymbol{\psi}]]=\int_{\partial \Omega} \mathcal{S}[\boldsymbol{\psi}] \cdot \mathcal{T}^{+}[\boldsymbol{\psi}]=0 \\
& \int_{\complement \Omega} \pi[\nabla \boldsymbol{v}[\boldsymbol{\psi}]]=-\int_{\partial \Omega} \mathcal{S}[\boldsymbol{\psi}] \cdot \mathcal{T}^{-}[\boldsymbol{\psi}]=0 .
\end{aligned}
$$

Hence by Lemma 1 it follows that $\boldsymbol{v}[\boldsymbol{\psi}]=\mathbf{0}$ in $\mathbb{R}^{3}$ so that by (27) $\boldsymbol{\psi}=\mathbf{0}$.

Let $\psi \in \operatorname{Kern} \mathcal{S}^{\prime}$ and let $\left\{\boldsymbol{\psi}_{k}\right\}_{k \in \mathbb{N}}$ be a regular sequence which converges to $\psi$ strongly in $W^{1-k-1 / q^{\prime}, q^{\prime}}(\partial \Omega)$. Of course, from (24) it follows that $v\left[\boldsymbol{\psi}_{k}\right] \rightarrow \boldsymbol{v}[\boldsymbol{\psi}]$ strongly in $W^{2-k, q^{\prime}}(\Omega)$. Let $z$ be the solution of

$$
\begin{aligned}
\operatorname{div} \mathbb{C}[\nabla z]=\boldsymbol{\phi} & \text { in } \Omega, \\
\boldsymbol{z}=\mathbf{0} & \text { on } \partial \Omega .
\end{aligned}
$$

Then, integrating by parts we have

$$
\int_{\Omega} v\left[\psi_{k}\right] \cdot \boldsymbol{\phi}=\int_{\partial \Omega} \mathcal{S}\left[\psi_{k}\right] \cdot \boldsymbol{s}(z)
$$

Hence letting $k \rightarrow+\infty$ it follows that

$$
\int_{\Omega}^{\star} v[\psi] \cdot \phi=0
$$

for all $\phi \in C_{0}^{\infty}(\Omega)$ so that $v[\psi]=0$ in $\Omega$.

It is well-known that the system

$$
\begin{aligned}
& \operatorname{div} \mathbb{C}[\nabla z]=\boldsymbol{\phi} \quad \text { in } \complement \bar{\Omega}, \\
& z=\mathbf{0} \quad \text { on } \partial \Omega
\end{aligned}
$$

has a unique solution $z \in D_{0}^{1,2}(\Omega)$. Let $g$ be a regular function vanishing outside $S_{2 R}$, equal to 1 in $S_{R}$ and such that $|\nabla g| \leq c / R$ for $R \gg \operatorname{diam} \Omega$. Then integrating by parts we have

$$
\begin{aligned}
\int_{\complement \Omega} g \boldsymbol{v}\left[\boldsymbol{\psi}_{k}\right] \cdot \boldsymbol{\phi} & =-\int_{\partial \Omega} \mathcal{S}\left[\boldsymbol{\psi}_{k}\right] \cdot \boldsymbol{s}(\boldsymbol{z}) \\
& +\int_{\complement \Omega} \nabla g \cdot\left\{\mathbb{C}\left[\nabla \boldsymbol{v}\left[\boldsymbol{\psi}_{k}\right]\right] \boldsymbol{z}-\mathbb{C}[\nabla \boldsymbol{z}] \boldsymbol{v}\left[\boldsymbol{\psi}_{k}\right]\right\} .
\end{aligned}
$$


By Schwarz's inequality and the properties of $g$

$$
\begin{aligned}
\left|\int_{C \Omega} \nabla g \cdot \mathbb{C}\left[\nabla \boldsymbol{v}\left[\boldsymbol{\psi}_{k}\right]\right] \boldsymbol{z}\right| & \leq \frac{c}{R} \int_{S_{2 R} \backslash S_{R}}\left|\nabla \boldsymbol{v}\left[\boldsymbol{\psi}_{k}\right]\right||\boldsymbol{z}| \\
& \leq \frac{c}{R}\left\|\nabla \boldsymbol{v}\left[\boldsymbol{\psi}_{k}\right]\right\|_{L^{2}\left(S_{2 R} \backslash S_{R}\right)}\|\boldsymbol{z}\|_{L^{2}\left(S_{2 R} \backslash S_{R}\right)}
\end{aligned}
$$

Likewise,

$$
\left|\int_{\mathbb{C} \Omega} \nabla g \cdot \mathbb{C}[\nabla \boldsymbol{z}] \boldsymbol{v}\left[\boldsymbol{\psi}_{k}\right]\right| \leq \frac{c}{R}\|[\nabla \boldsymbol{z}]\|_{L^{2}\left(S_{2 R} \backslash S_{R}\right)}\left\|\boldsymbol{v}\left[\boldsymbol{\psi}_{k}\right]\right\|_{L^{2}\left(S_{2 R} \backslash S_{R}\right)} .
$$

Therefore, taking into account Hardy's inequality

$$
\int_{C \Omega} \frac{|\boldsymbol{h}|^{2}}{r^{2}} \leq c \int_{\complement \Omega}|\nabla \boldsymbol{h}|^{2}
$$

for all $h \in D_{0}^{1,2}(\Omega)$, we can let $R \rightarrow+\infty$ in (36) to have

$$
\int_{\complement \Omega} v\left[\psi_{k}\right] \cdot \boldsymbol{\phi}=-\int_{\partial \Omega} \mathcal{S}\left[\psi_{k}\right] \cdot s(z) .
$$

Hence letting $k \rightarrow+\infty$ yields

$$
\int_{\complement \Omega}^{\star} v[\psi] \cdot \phi=0
$$

so that $v[\psi]=\mathbf{0}$ in $\complement \Omega$ and (27) and the above results imply that $\boldsymbol{\psi}=\mathbf{0}$.

The proof of the Lemma for $\Omega$ exterior follows the same steps so it is omitted.

\section{The Trace Operators Associated with the Double Layer Potential}

For every $\varphi \in L^{1}(\partial \Omega)$ the field

$$
\boldsymbol{w}[\boldsymbol{\varphi}](x)=\int_{\partial \Omega} \mathbb{C}[\nabla \boldsymbol{U}(x-\zeta)](\boldsymbol{\varphi} \otimes \boldsymbol{n})(\zeta) \mathrm{d} \sigma_{\zeta}
$$

defines analytical solutions of (1) in $\mathbb{R}^{3} \backslash \partial \Omega$ and is known as double layer potential with density $\varphi$. Note that

$$
\nabla_{k} \boldsymbol{w}[\boldsymbol{\varphi}](x)=O\left(r^{-2-k}\right) .
$$

The trace on $\partial \Omega$ of a double layer potential with density $\varphi \in W^{k-1 / q, q}(\partial \Omega)$ is defined on both "faces" of $\partial \Omega$ by the limit

$$
\lim _{\epsilon \rightarrow 0^{+}} \boldsymbol{w}[\boldsymbol{\varphi}](\xi \mp \epsilon \boldsymbol{l}(\xi))=\mathcal{W}^{ \pm}[\boldsymbol{\psi}](\xi)
$$

for almost all $\xi \in \partial \Omega$ (by the embedding theorem if $q>3 / k$, then (40) holds for all $\xi$ ) and axis $l$ in a ball tangent (on the side of $n$ ) to $\partial \Omega$ at $\xi$. Moreover,

$$
\left\|\mathcal{W}^{ \pm}[\boldsymbol{\varphi}]\right\|_{W^{k-1 / q, q}(\partial \Omega)} \leq c\|\varphi\|_{W^{k-1 / q, q}(\partial \Omega)}
$$

and for $\Omega$ bounded

$$
\|\boldsymbol{w}[\boldsymbol{\varphi}]\|_{W^{k, q}(\Omega)} \leq c\|\boldsymbol{\varphi}\|_{W^{k-1 / q, q}(\partial \Omega)}
$$

for some constant $c$ depending only on $k, q$ and $\Omega$. The jump condition

$$
\boldsymbol{\varphi}=\mathcal{W}^{+}[\varphi]-\mathcal{W}^{-}[\varphi]
$$


holds and the classical Liapounov-Tauber theorem assures that the traction field associated with $w[\varphi]$ assumes the same value on both "faces" of $\partial \Omega$

$$
\lim _{\epsilon \rightarrow 0^{ \pm}} \mathbb{C}[\nabla \boldsymbol{w}[\boldsymbol{\varphi}]](\xi-\epsilon \boldsymbol{l}(\xi)) \boldsymbol{n}(\xi)=\mathcal{Z}[\boldsymbol{\varphi}](\xi)
$$

and defines a linear, continuous operator

$$
\mathcal{Z}: W^{k-1 / q, q}(\partial \Omega) \rightarrow W^{k-1-1 / q, q}(\partial \Omega)
$$

i.e.,

$$
\|\mathcal{Z}[\boldsymbol{\varphi}]\|_{W^{k-1-1 / q, q(\partial \Omega)}} \leq c\|\boldsymbol{\varphi}\|_{W^{k-1 / q, q(\partial \Omega)}}
$$

for some constant $c$ depending only on $k, q$ and $\Omega$. A standard argument shows that $\mathcal{W}^{ \pm}$and $\mathcal{T}^{\mp}$ are adjoint each other. Hence, for instance,

$$
\mathcal{W}^{-}: W^{2-k-1 / q^{\prime}, q^{\prime}}(\partial \Omega) \rightarrow W^{2-k-1 / q^{\prime}, q^{\prime}}(\partial \Omega)
$$

is the adjoint of

$$
\mathcal{T}^{+}: W^{k-1-1 / q, q}(\partial \Omega) \rightarrow W^{k-1-1 / q, q}(\partial \Omega)
$$

and defines the trace of a double layer potential $w[\varphi]$ with density in $W^{2-k-1 / q^{\prime}, q^{\prime}}(\partial \Omega)$ :

$$
\boldsymbol{w}[\boldsymbol{\varphi}](x)=\int_{\partial \Omega}^{\star} \mathbb{C}[\nabla \boldsymbol{U}(x-\zeta)](\boldsymbol{\varphi} \otimes \boldsymbol{n})(\zeta) \mathrm{d} \sigma_{\zeta} .
$$

As we did for the trace operator of the single layer potential we can show that the adjoint operator of $\mathcal{Z}$

$$
\mathcal{Z}^{\prime}: W^{2-k-1 / q^{\prime}, q^{\prime}}(\partial \Omega) \rightarrow W^{1-k-1 / q^{\prime}, q^{\prime}}(\partial \Omega)
$$

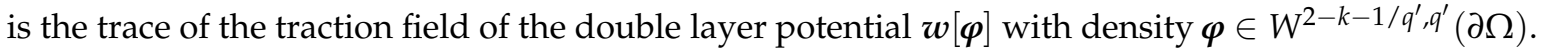

As for the operator $\mathcal{S}$ in the previous section, starting from Lemma 3, 4, we show that the operators $\mathcal{W}^{ \pm}, \mathcal{T}^{ \pm}$and $\mathcal{Z}$ are Fredholmian.

Theorem 2. Let $\Omega$ be a bounded or an exterior domain of class $C^{k}(k \geq 2)$. The operators $\mathcal{W}^{ \pm}, \mathcal{T}^{ \pm}$are Fredholmian, $\operatorname{Kern} \mathcal{W}^{+}=\operatorname{Kern} \mathcal{T}^{-}=\{\mathbf{0}\}$ and

$$
\begin{aligned}
\text { Kern } \mathcal{T}^{+} & = \begin{cases}\{\boldsymbol{\psi}: \mathcal{S}[\boldsymbol{\psi}] \in \mathfrak{R}\}, & \Omega \text { bounded, } \\
\{\mathbf{0}\}, & \Omega \text { exterior, }\end{cases} \\
\text { Kern } \mathcal{W}^{-} & = \begin{cases}\mathfrak{R}, & \Omega \text { bounded, } \\
\{\mathbf{0}\}, & \Omega \text { exterior. }\end{cases}
\end{aligned}
$$


Proof. Let $\Omega$ be bounded. By Lemmas 3 and 4 , the trace theorem and interior estimates

$$
\begin{gathered}
\left\|\mathcal{W}^{+}[\boldsymbol{\varphi}]\right\|_{W^{k-1 / q, q}(\partial \Omega)} \leq\|\boldsymbol{w}[\boldsymbol{\varphi}]\|_{W^{k, q}(\Omega)} \leq c\|\mathcal{Z}[\boldsymbol{\varphi}]\|_{W^{k-1-1 / q, q}(\partial \Omega)} \\
\leq c\|\boldsymbol{w}[\boldsymbol{\varphi}]\|_{W^{k, q}\left(\mathcal{C} \Omega \cap S_{R}\right)} \leq c\left\{\left\|\mathcal{W}^{-}[\boldsymbol{\varphi}]\right\|_{W^{k-1 / q, q}(\partial \Omega)}+\|\mathcal{C}\|\right\} \\
\left\|\mathcal{W}^{-}[\boldsymbol{\varphi}]\right\|_{W^{k-1 / q, q}(\partial \Omega)} \leq\|\boldsymbol{w}[\boldsymbol{\varphi}]\|_{W^{k, q}\left(\bar{\Omega} \cap S_{R}\right)} \leq c\left\{\|\mathcal{Z}[\boldsymbol{\varphi}]\|_{W^{k-1-1 / q, q}(\partial \Omega)}+\left\|\mathcal{C}_{1}\right\|\right\} \\
\leq c\left\{\left\|\mathcal{W}^{+}[\boldsymbol{\varphi}]\right\|_{W^{k-1 / q, q}(\partial \Omega)}+\left\|\mathcal{C}_{1}\right\|\right\} \\
\left\|\mathcal{T}^{+}[\boldsymbol{\psi}]\right\|_{W^{k-1-1 / q, q}(\partial \Omega)} \leq\|\boldsymbol{v}[\boldsymbol{\psi}]\|_{W^{k, q}(\Omega)} \leq c\|\mathcal{S}[\boldsymbol{\psi}]\|_{W^{k-1 / q, q}(\partial \Omega)} \\
\leq c\|\boldsymbol{v}[\boldsymbol{\psi}]\|_{W^{k, q}\left(\mathcal{C} \Omega \bar{\Omega}_{R}\right)} \leq c\left\{\left\|\mathcal{T}^{-}[\boldsymbol{\psi}]\right\|_{W^{k-1-1 / q, q}(\partial \Omega)}+\left\|\mathcal{C}_{2}\right\|\right\} \\
\left\|\mathcal{T}^{-}[\boldsymbol{\psi}]\right\|_{W^{k-1-1 / q, q}(\partial \Omega)} \leq\|\boldsymbol{v}[\boldsymbol{\psi}]\|_{W^{k, q}\left(\complement \bar{\Omega} \cap S_{R}\right)} \leq c\left\{\|\mathcal{S}[\boldsymbol{\psi}]\|_{W^{k-1 / q, q}(\partial \Omega)}+\left\|\mathcal{C}_{3}\right\|\right\} \\
\leq c\left\{\left\|\mathcal{T}^{+}[\boldsymbol{\psi}]\right\|_{W^{k-1-1 / q, q}(\partial \Omega)}+\left\|\mathcal{C}_{3}\right\|\right\}
\end{gathered}
$$

where $\mathcal{C}, \mathcal{C}_{1}$ are completely continuous maps from $W^{k-1 / q, q}(\partial \Omega)$ in a Banach space and $\mathcal{C}_{2}, \mathcal{C}_{3}$ completely continuous maps from $W^{k-1-1 / q, q}(\partial \Omega)$ in a Banach space. Therefore, by (42), (43) and (27)

$$
\begin{aligned}
& \|\boldsymbol{\varphi}\|_{W^{k-1 / q, q}(\partial \Omega)} \leq c\left\{\left\|\mathcal{W}^{ \pm}[\boldsymbol{\varphi}]\right\|_{W^{k-1 / q, q}(\partial \Omega)}+\left\|\mathcal{C}^{\prime}\right\|\right\} \\
& \|\boldsymbol{\psi}\|_{W^{k-1-1 / q, q(\partial \Omega)}} \leq c\left\{\left\|\mathcal{T}^{ \pm}[\boldsymbol{\psi}]\right\|_{W^{k-1-1 / q, q}(\partial \Omega)}+\left\|\mathcal{C}^{\prime \prime}\right\|\right\}
\end{aligned}
$$

for some completely continuous operators. Hence it follows that $\mathcal{W}^{ \pm}$and $\mathcal{T}^{ \pm}$have closed ranges.

If $\varphi \in \operatorname{Kern} \mathcal{W}^{+}$. By the uniqueness theorem $\boldsymbol{w}[\boldsymbol{\varphi}]=\mathbf{0}$ in $\Omega$ so that $\mathcal{Z}[\boldsymbol{\varphi}]=\mathbf{0}$. Hence again by uniqueness $w[\varphi]=\mathbf{0}$ in $\complement \Omega$ so that $\varphi=0$ on $\partial \Omega$. If $\psi \in \operatorname{Kern} \mathcal{T}^{-}$, with $\mathcal{T}^{-}: W^{1-k-1 / q^{\prime}, q^{\prime}}(\partial \Omega) \rightarrow$ $W^{1-k-1 / q^{\prime}, q^{\prime}}(\partial \Omega)$, consider a regular sequence $\psi_{k}$ which converges to $\psi$ strongly in $W^{1-k-1 / q^{\prime}, q^{\prime}}(\partial \Omega)$ and the solution $z$ of

$$
\begin{aligned}
\operatorname{div} \mathbb{C}[\nabla z] & =\boldsymbol{\phi} & & \text { in } \complement \bar{\Omega}, \\
\boldsymbol{s}(\boldsymbol{z}) & =\mathbf{0} & & \text { on } \partial \Omega
\end{aligned}
$$

for $\phi \in C_{0}^{\infty}(\Omega)$. Integrating by parts and taking into account (17), we have

$$
\int_{C \Omega} v\left[\psi_{k}\right] \cdot \phi=-\int_{\partial \Omega} z \cdot \mathcal{T}^{-}\left[v\left[\psi_{k}\right]\right.
$$

Hence, letting $k \rightarrow+\infty$, it follows that $v[\psi]=0$ in $\complement \Omega$, so that $\mathcal{T}^{-}[\boldsymbol{\psi}]=0$. On the other hand, by uniqueness $v[\boldsymbol{\psi}]=0$ in $\Omega$ so that $\mathcal{T}^{+}[\boldsymbol{\psi}]=\mathbf{0}$. Hence by (27) $\boldsymbol{\psi}=\mathbf{0}$. The proof of the other properties are quite analogous so it is omitted.

Theorem 3. Let $\Omega$ be a bounded domain or an exterior domain of class $C^{k}(k \geq 2)$. The operator $\mathcal{Z}$ is Fredholmian and

$$
\operatorname{Kern} \mathcal{Z}=\operatorname{Kern} \mathcal{Z}^{\prime}=\mathfrak{R}
$$

Proof. Let $\Omega$ be bounded. The trace theorem and interior estimates yield

$$
\begin{aligned}
\|\boldsymbol{\varphi}\|_{W^{k-1 / q, q}(\partial \Omega)} & \leq\left\|\mathcal{W}^{+}[\boldsymbol{\varphi}]\right\|_{W^{k-1 / q, q}(\partial \Omega)}+\left\|\mathcal{W}^{-}[\boldsymbol{\varphi}]\right\|_{W^{k-1 / q, q}(\partial \Omega)} \\
& \leq c\left\{\|\boldsymbol{w}[\boldsymbol{\varphi}]\|_{W^{k, q}(\Omega)}+\|\boldsymbol{w}[\boldsymbol{\varphi}]\|_{W^{k, q}\left(C \Omega \cap S_{R}\right)}\right\} \\
& \leq c\left\{\| \mathcal{Z}\left[\boldsymbol{\varphi}\left\|_{W^{k-1-1 / q, q}(\partial \Omega)}+\right\| \mathcal{C} \|\right\},\right.
\end{aligned}
$$


with $\mathcal{C}$ completely continuous map from $W^{k-1 / q, q}(\partial \Omega)$ in a Banach space. Therefore, by Peetre's result, $\mathcal{Z}$ has closed range.

Let $\varphi \in \operatorname{Kern} \mathcal{Z}$. By the uniqueness theorem $w[\varphi]=0$ in $\complement \Omega$ and $w[\varphi] \in \Re$ in $\Omega$. Therefore, by (43), it follows that $\varphi \in \Re$. On the other hand, a direct inspection shows that $\varphi \in \mathfrak{R}$ belongs to Kern $\mathcal{Z}$.

Let now $\varphi \in \operatorname{Kern} \mathcal{Z}^{\prime}$ and consider the sequence $\varphi_{k}$ strongly converging to $\varphi$ in $W^{2-k-1 / q, q}(\partial \Omega)$ and the solution $z$ of

$$
\begin{aligned}
\operatorname{div} \mathbb{C}[\nabla z] & =\boldsymbol{\phi} & & \text { in } \Omega, \\
\boldsymbol{s}(z) & =\mathbf{0} & & \text { on } \partial \Omega
\end{aligned}
$$

with $\phi$ such that

$$
\int_{\Omega} \varrho \cdot \phi=0, \quad \forall \varrho \in \mathfrak{R}
$$

An integration by parts yields

$$
\int_{\Omega} w\left[\varphi_{k}\right] \cdot \phi=-\int_{\partial \Omega} z \cdot s\left(w\left[\varphi_{k}\right]\right) .
$$

Hence, letting $k \rightarrow+\infty$, it follows that $\boldsymbol{w}[\boldsymbol{\varphi}] \in \mathfrak{R}$ in $\Omega$ so that, by (43), Kern $\mathcal{Z}^{\prime}=\operatorname{Kern} \mathcal{Z}=\mathfrak{R}$. The proof for exterior domains is analogous to the previous one, so it is omitted.

\section{Conclusions}

In this article we dealt with some properties related to the trace operators associated with the elastic layer potentials. In particular, we proved that their extensions to some sets of singular densities satisfy the Fredholm property (Theorems 1-3). These results represent an important step in the analysis of the system of linear elastostatics, as they could lead to the existence and uniqueness of solutions to the main boundary value problems with singular data, to which we are going to dedicate our next researches.

Author Contributions: The authors contributed equally to this work. All authors read and approved the final manuscript.

Funding: This research was supported by Programma VALERE - Università degli Studi della Campania "Luigi Vanvitelli".

Conflicts of Interest: The authors declare no conflict of interest.

\section{References}

1. Gurtin, M.E. The linear theory of elasticity. in Handbuch der Physik; Truesedell, C., Ed.; Springer-Verlag: Berlin/Heidelberg, Germany, 1972.

2. Fichera, G. Sull'esistenza e sul calcolo delle soluzioni dei problemi al contorno, relativi all'equilibrio di un corpo elastico. Annali della Scuola Normale Superiore di Pisa 1950, 4, 35-99.

3. Fichera, G. Existence theorems in elasticity. In Handbuch der Physik; Truesedell, C., Ed.; Springer-Verlag: Berlin/Heidelberg, Germany, 1972.

4. Russo, R. An extension of the basic theorems of linear elastostatics to exterior domains. Ann. Univ. Ferrara, Sez. VII Sci. Mat. 1988, 34, 101-119.

5. Russo, R. On the traction problem in linear elastostatics. J. Elast. 1992, 27, 57-68. [CrossRef]

6. Kupradze, V.D.; Gegelia, T.G.; Basheleishvili, M.O.; Burchuladze, T.V. Three Dimensional Problems of the Mathematical Theory of Elasticity and Thermoelasticity; North-Holland: Amsterdam, The Netherlands, 1979.

7. John, F. Plane Waves and Spherical Means Applied to Partial Differential Equations; Interscience: New York, NY, USA, 1955.

8. Duvant, G.; Lions, J.L. Inequalities in Mechanics and Physics; Springer-Verlag: Berlin/Heidelberg, Germany, 1976. 
9. Russo, R. On Stokes' problem. In Advances in Mathematical Fluid Mechanics; Rannacher, R., Sequeira, A., Eds.; Springer-Verlag: Berlin/Heidelberg, Germany, 2010; pp. 473-511.

10. Van Hove, L. Sur l'extension de la condition de Legendre du calcul des variations aux intégrales multiples à plusieurs fonctions inconnues. Proc. Koninkl. Ned. Adad. Wetenschap. 1947, 50, 18-23.

11. Giusti, E. Direct Methods in the Calculus of Variations; Word Scientific: Singapore, 2004.

12. Schechter, M. Principles of Functional Analysis; Graduate Studies in Mathematics; American Mathematical Society: Providence, RI, USA, 2002.

13. Miranda, C. Partial Differential Equations of Elliptic Type; Springer-Verlag: Berlin/Heidelberg, Germany, 1970.

14. Lions, J.L. Magenes, E. Non-Homogeneous Boundary-Value Problems and Applications; Springer-Verlag: Berlin/Heidelberg, Germany, 1972; Volume I.

15. Nečas, J. Les Méthodes Directes en Théorie des Équations Élliptiques; Masson: Paris, France; Academie: Prague, Czech Republic, 1967.

16. Russo, A.; Tartaglione, A. Strong uniqueness theorems and the Phragmen-Lindelof principle in nonhomogeneous elastostatics. J. Elast. 2011, 102, 133-149. [CrossRef]

17. Russo, A.; Tartaglione, A. On the contact problem of classical elasticity. J. Elast. 2010, 99, 19-38. [CrossRef]

18. Tartaglione, A. On existence, uniqueness and the maximum modulus theorem in plane linear elastostatics for exterior domains. Ann. Univ. Ferrara, Sez. VII Sci. Mat. 2001, 47, 89-106.

(C) 2019 by the authors. Licensee MDPI, Basel, Switzerland. This article is an open access article distributed under the terms and conditions of the Creative Commons Attribution (CC BY) license (http:/ / creativecommons.org/licenses/by/4.0/). 